MPP-2010-21

\title{
Basics of F-theory from the Type IIB Perspective
}

\author{
Ralph Blumenhagen ${ }^{1,}$ \\ ${ }^{1}$ Max-Planck-Institut für Physik, Föhringer Ring 6, 80805 München, Germany
}

These short lecture notes provide an introduction to some basic notions of F-theory with some special emphasis on its relation to Type IIB orientifolds with O7/O3-planes.

Copyright line will be provided by the publisher

\section{Introduction}

Historically outstripping heterotic string compactifications and intersecting D-brane models, the last two years have seen the main activity in the field of string phenomenology shifting towards F-theory models. In this framework some of the model building shortcomings of D-brane realizations of grand unified theories (GUTs) can be overcome.

To appreciate this, let us recall some issues on D-brane constructions. The gauge theories are supported on D-branes, which in general can have a dimension smaller than the bulk. Completely occupying our observable large scale four-dimensional world, they wrap certain sub-manifolds of the internal geometry. The matter fields are localized on the intersections of such D-branes. Since the early years of so-called intersecting D-brane models, it was clear that this set-up naturally allows for semi-simple gauge groups with matter fields in the bifundamental representations. Therefore, here one directly engineers the $S U(3)_{c} \times S U(2)_{w} \times U(1)_{Y}$ MSSM, while leaving the unification of gauge couplings at the GUT scale essentially unexplained. Indeed, the gauge couplings depend on the generally different volumes of the internal cycles wrapped by the D-branes supporting each gauge factor.

It was quickly realized that the construction of GUT groups $S O(10)$ and $S U(5)$ was obstructed by the perturbative absence of matter fields in the 16 representation of $S O(10)$ respectively by the absence of the top Yukawa coupling $\mathbf{1 0 1 0} \mathbf{5}_{\mathbf{H}}$ for the $S U(5)$ case. These two latter features are of non-perturbative origin for orientifold models (see [1] for a review). It was realized two years ago that the aforementioned problems with realizing simple GUT groups in orientifold constructions are nicely reconciled in F-theory models on elliptically fibered Calabi-Yau four-folds [2]-5] (see [6] for a more phenomenological review).

One can think of F-theory as Type IIB compactifications on compact complex n-dimensional manifolds $B_{n}$ with general $(p, q) 7$-branes wrapping $2(n-1)$ cycles of $B_{n}$. Since the 7-branes are of real co-dimension two, the solutions to the Laplace equations are of logarithmic type. Therefore, the backreaction of the 7-branes on the geometry and the dilaton is always substantial and has to be taken into account. By identifying the strong-weak $S L(2, \mathbb{Z})$ duality of the Type IIB superstring with the modular group of a torus, C. Vafa [7] showed that the backreaction can geometrically be taken into account by an elliptic fibration over the base $B_{n}$, where the modular parameter of the fiber is identified with the axio-dilaton field of Type IIB. The location of the 7-branes correspond to the degeneration loci of the elliptic fibration and for supersymmetry the fibrations have to be of Calabi-Yau type.

Due to the strong backreaction, only in a global $g_{s} \rightarrow 0$ limit a general F-theory model is expected to correspond to an orientifold. F-theory inherently contains some features which are non-perturbative from the orientifold point of view. This is the reason for the appearance of exceptional groups in F-theory, which by a further breaking also realize the spinor representation of a GUT $S O(10)$ as well as the top-quark Yukawa couplings $\mathbf{1 0} \mathbf{1 0} \mathbf{5}_{\mathbf{H}}$ in GUT $S U(5)$. For four-dimensional models, the basis $B_{3}$ is a Fano three-fold and the 7-branes wrap complex surfaces, i.e. four-cycle.

Thus, F-theory is a non-perturbative completion of Type IIB orientifolds where the 7-branes are completely encoded in the geometry of the elliptic fibration. The aim of this lecture is to give an introduction 
into some basis notions of F-theory, which essentially addresses those students, who are already familiar with D-brane constructions. It is explained why F-theory is inevitable for the correct study of Type IIB compactifications with D7-branes, in which sense it goes beyond the perturbative Type IIB superstring and how this leads to a solution of the above mentioned problems with GUT models. Note that this was the first of a series of two lectures on F-theory GUTs held at the 9th Hellenic School on Elementary Particle Physics and Gravity, Corfu 2009. The second lecture focused more on the specifics of realizing four-dimensional $S U(5)$ GUTs from F-theory. Please consult [8] for more detailed lecture notes on F-theory.

\section{$2 \quad$ D7-branes and $S L(2, \mathbb{Z})$ self-duality}

As a starting point, we consider Type IIB orientifolds compactified on a Calabi-Yau three-fold $X$ and an orientifold projection $\Omega \sigma(-1)^{F_{L}}$ (see [9] for a review). Here $\sigma$ denotes a holomorphic involution of $X$ acting as

$$
\sigma^{*}(J)=J, \quad \sigma^{*}\left(\Omega_{3}\right)=-\Omega_{3}
$$

on the Kähler respectively holomorphic (3,0)-form of $X$. This orientifold quotient introduces an O7-plane into the theory, whose tadpole is canceled by the introduction of stacks of D7-branes wrapping various holomorphic four-cycles of $X$, whose total homology class in $H_{4}(X, \mathbb{Z})$ is equal to the one of the 07 plane. One can now compute the (chiral) massless spectrum coming from the lowest excitations of open strings stretched between various pairs of D7-branes. This gives rise to gauge bosons of only unitary or orthogonal/symplectic gauge groups and in addition to matter fields transforming solely in bifundamental or (anti)-symmetric representations of the gauge group. This is simply a consequence of the fact that an open string has two ends. Clearly, such open string excitations can never give rise to exceptional gauge groups and, as a group theoretic consequence, to matter in the spinor representation of an $S O(10)$ gauge group.

What we have just briefly described is the construction of perturbative Type IIB orientifold string vacua and its short-comings when it comes to GUT like structures. Naively, this seems to be the end of the story. However, taking the perturbative string limit $g_{s} \ll 1$ is, to say the least, quite questionable, if branes of (real) co-dimension two, such as D7-branes, are present. This becomes evident by studying the D7-brane solution in Type IIB supergravity [10], which is magnetically charged under the R-R scalar field $C_{0}$ with corresponding field strength $F_{1}=d C_{0}$.

The space transverse to the D7-brane is two-dimensional so that it is convenient to combine the two transverse coordinates into a single complex variable $z=y^{1}+i y^{2}$. Furthermore ones combine the Type IIB dilaton together with the R-R scalar field $C_{0}$ into a complex scalar field $\tau=C_{0}+i e^{-\Phi}$. For the D7brane supergravity solution, $\tau$ will be a function of the complex coordinate $z$, and the field equation, i.e. the Laplace equation in two-dimensions, is now written as $\partial_{\bar{z}} \tau(z, \bar{z})=0$. This means that $\tau$ must be a holomorphic function. However not any holomorphic solution is a good solution, e.g. we must require $\operatorname{Im} \tau>0$. Furthermore the solution must have finite energy per unit volume, and it turns out that for this purpose one needs the $S L(2, \mathbb{Z})$ action on $\tau$. The solution can then be written as $j(\tau)=z^{-1}$, where

$$
j(\tau)=\frac{\left(\vartheta_{3}^{8}(\tau)+\vartheta_{4}^{8}(\tau)+\vartheta_{2}^{8}(\tau)\right)^{3}}{8 \eta^{24}(\tau)}=e^{-2 \pi i \tau}+744+196884 e^{2 \pi i \tau}+\ldots
$$

is the modular invariant $j$-function. Close to the D7-brane, i.e. at $|z| \rightarrow 0$ this solution behaves as

$$
\tau(z) \sim \frac{1}{2 \pi i} \log z
$$

which, circling once around the origin, gives rise to a monodromy $\tau \rightarrow \tau+1$. This monodromy reflects that the D7-brane carries $C_{0}$ charge one. Due to this logarithmic dependence of the axio-dilaton on the transverse coordinate $z$, the backreaction of the D7-brane is so strong that one cannot really control the 
weak-coupling regime. Once the string coupling is non-zero and maybe small somewhere, it necessarily becomes large in other regions of the transverse space. However, eq.(3) implies that $g_{s}=\exp (\Phi)$ is small close to the D7-brane so that for the gauge theory on the D7-brane we expect a weak coupling description.

As will now review, also the backreaction on the metric is strong. Consider the 10-dimensional spacetime metric of the D7-brane solution: $\mathrm{d} s^{2}=-\mathrm{d} t^{2}+\sum_{i=1}^{7} \mathrm{~d} x_{i}^{2}+e^{B(z, \bar{z})} \mathrm{d} z \mathrm{~d} \bar{z}$. Then the Einstein equation connects the warp factor $B$ with the dilaton field, and one obtains the following simple relation $\partial \bar{\partial} B=$ $\partial \bar{\partial} \log (\operatorname{Im} \tau)$. It turns out that the solution with the correct modular properties and the right asymptotic behavior far away from the D7-brane is given by

$$
e^{B(z, \bar{z})}=(\operatorname{Im} \tau) \frac{\eta^{2}(\tau) \bar{\eta}^{2}(\bar{\tau})}{\left|\prod_{i=1}^{N}\left(z-z_{i}\right)\right|^{\frac{1}{6}}} .
$$

Here $N$ is the number of 7-branes and the $z_{i}$ denote their positions in the two-dimensional transverse space. Expanding this function for large $|z|$ one gets that $B(z, \bar{z}) \sim-N / 12 \cdot \log |z|$. Using this asymptotic behavior one realizes that the metric goes like $\left|z^{-N / 12} \mathrm{~d} z\right|^{2}$ far away from the 7-branes. This means that each 7-brane leaves a deficit angle of $2 \pi / 12$ in the transverse space. In fact precisely 247 -branes are required to get the deficit angle $4 \pi$ of the compact two-dimensional sphere $\mathbb{C P}^{1}$. Therefore, there exists a supersymmetric compactification of the Type IIB superstring on $\mathbb{C P}^{1}$ with precisely 24 7-branes and a varying dilaton.

So far we have only been talking about D7-branes. However, due to the $S L(2, \mathbb{Z})$ duality symmetry there exist infinitely many different kinds of 7-branes. Indeed, since there exists a doublet of two-forms $\left(B_{2}, C_{2}\right)$ in the ten-dimensional Type IIB string theory, there are not only fundamental strings and D1branes, but also strings carrying electric charges $(p, q)$, where in this notation a fundamental string is a $(1,0)$ string. Acting with an $S L(2, \mathbb{Z})$ transformation on the fundamental string gives

$$
\left(\begin{array}{l}
p \\
q
\end{array}\right)=\left(\begin{array}{ll}
p & r \\
q & s
\end{array}\right)\left(\begin{array}{l}
1 \\
0
\end{array}\right) \quad \text { with } p s-q r=1 .
$$

Similarly, one has a doublet of ten-dimensional scalars $\left(C_{0}, \Phi\right)$ leading to magnetically charged $(p, q) 7$ branes, where a D7-brane (charged only under $\left.C_{0}\right)$ is a $(1,0)$ 7-brane. Since a fundamental string can end on a D7-brane, $S L(2, \mathbb{Z})$ implies that a $(p, q)$ string can end on a $(p, q) 7$-brane. In eq. (3) we have seen that the solitonic solution of a D7-brane induces an $\operatorname{SL}(2, \mathbb{Z})$ monodromy $M_{\mathrm{D} 7}=\left(\begin{array}{ll}1 & 1 \\ 0 & 1\end{array}\right)$. Applying the $S L(2, \mathbb{Z})$ symmetry, a general $(p, q) 7$-brane induces a monodromy

$$
M_{(p, q)}=\left(\begin{array}{ll}
p & r \\
q & s
\end{array}\right)\left(\begin{array}{ll}
1 & 1 \\
0 & 1
\end{array}\right)\left(\begin{array}{ll}
p & r \\
q & s
\end{array}\right)^{-1}=\left(\begin{array}{cc}
1-p q & p^{2} \\
-q^{2} & 1+p q
\end{array}\right)
$$

For later purpose we consider the three 7-branes $A=(1,0), B=(1,-1)$ and $C=(1,1)$. It is now straightforward to compute the monodromy matrices for the combinations of these three 7-branes listed in table 1. To understand the massless modes between such more general $(p, q)$ 7-branes, one notices that the $(p, q)$-strings can form so-called string junctions. For instance a $(1,1)$ string can split into a $(1,0)$ and $(0,1)$ string. Similar to open $(1,0)$ strings ending on D7-branes, for more general 7-brane there can exist so-called string junctions ending on them. For instance there can be a string junction with four external strings of type $(1,0)-(1,0)-(1,1)-(1,-1)$, which can end on the respective 7-branes $A-A-B-C$. Clearly, such objects can give qualitatively new massless states beyond what is possible with perturbative fundamental open strings. We will come back to this in the next section.

\section{F-theory}

The observations made in the previous section led C. Vafa in 1996 to the idea of F-theory. This is a hypothetical or rather auxiliary twelve dimensional theory which, when compactified on a two-dimensional torus, gives the Type IIB superstring. The modular group of the torus is identified with the $S L(2, \mathbb{Z})$ symmetry of Type IIB. However, this twelve-dimensional interpretation is not meant in the sense of a standard 


\begin{tabular}{|c|c|c|}
\hline 7-branes & number & monodromies \\
\hline \hline$A$ & 1 & $M_{A}=\left(\begin{array}{ll}1 & 1 \\
0 & 1\end{array}\right)$ \\
\hline$B$ & 1 & $M_{B}=\left(\begin{array}{cc}2 & 1 \\
-1 & 0\end{array}\right)$ \\
\hline$C$ & 1 & $M_{C}=\left(\begin{array}{cc}0 & 1 \\
-1 & 2\end{array}\right)$ \\
\hline$A^{n}$ & $n$ & $M_{A}^{n}=\left(\begin{array}{ll}1 & n \\
0 & 1\end{array}\right)$ \\
\hline$A B$ & 2 & $M_{A} M_{B}=\left(\begin{array}{cc}1 & 1 \\
-1 & 0\end{array}\right)$ \\
\hline$A^{2} B$ & 3 & $M_{A}^{2} M_{B}=\left(\begin{array}{cc}0 & 1 \\
-1 & 0\end{array}\right)$ \\
\hline$A^{2} B A$ & 4 & $M_{A}^{2} M_{B} M_{A}=\left(\begin{array}{cc}0 & 1 \\
-1 & -1\end{array}\right)$ \\
\hline$A^{n} B C$ & $n+2$ & $M_{A}^{n} M_{B} M_{C}=\left(\begin{array}{cc}-1 & -n+4 \\
0 & -1\end{array}\right)$ \\
\hline$A^{5} B C B$ & 8 & $M_{A}^{5} M_{B} M_{C} M_{B}=\left(\begin{array}{cc}-1 & -1 \\
1 & 0\end{array}\right)$ \\
\hline$A^{6} B C B$ & 9 & $M_{A}^{6} M_{B} M_{C} M_{B}=\left(\begin{array}{cc}0 & -1 \\
1 & 0\end{array}\right)$ \\
\hline$A^{6} B C B A$ & 10 & $M_{A}^{6} M_{B} M_{C} M_{B} M_{A}=\left(\begin{array}{cc}0 & -1 \\
1 & 1\end{array}\right)$ \\
\hline
\end{tabular}

Table 1 Monodromies around stacks of 7-branes of types $A, B, C$.

Kaluza-Klein reduction, as first there does not exist a twelve dimensional supergravity theory with signature $(1,11)$ in the first place and second in ten dimensions there is no scalar field corresponding to the volume modulus of this $T^{2}$. Hence the 12-dimensional interpretation serves just to provide a geometrization of the Type IIB $S L(2, \mathbb{Z})$ duality symmetry rather than to correspond to a real compactification from twelve to ten dimensions. What makes true sense though is to start with the eleven-dimensional M-theory compactified on $T^{2}$ and define F-theory as the vol $\left(T^{2}\right) \rightarrow 0$ limit.

The true power of this F-theory picture reveals itself when compactifying the Type IIB superstring to lower dimensions. We have just recalled that there should exist a compactification of Type IIB on $\mathbb{C P}^{1}$ with 24 7-branes preserving half the supersymmetry, i.e. 16 supercharges. Observing that M-theory compactified on a $K 3$ surface breaks half the supersymmetry, one finds that F-theory compactified on an elliptically fibered $K 3$ with base $\mathbb{C P}^{1}$ is the Type IIB string compactified on the base $\mathbb{C P}^{1}$ with 24 7-branes.

For this to make sense, we have to find the 7-branes in this purely geometric description, where we recall that in Type IIB there exist not just ordinary D7-branes but also these $(p, q)$ 7-branes introduced in the previous section. We have seen that close to a D7-brane at position $u_{1} \in \mathbb{C P}^{1}$ the complexified dilaton behaves like $j(\tau) \simeq 1 /\left(u-u_{1}\right)$. Now $\tau$ is really the modular parameter of a geometric elliptic curve and it is known from mathematics that the $j$-function naturally appears in this context. For this purpose we explicitly write the elliptic curve as the hypersurface $\mathbb{P}_{1,2,3}[6]$ in the homogeneous coordinates $(z, x, y)$. The fibration over the base $B=\mathbb{C P}^{1}$ can then be written as the hypersurface constraint

$$
y^{2}+a_{1} x y z+a_{3} y z^{3}=x^{3}+a_{2} x^{2} z^{2}+a_{4} x z^{4}+a_{6} z^{6},
$$

where the coefficients $a_{n}$ are homogeneous polynomials of degree $2 n$ of the two homogeneous coordinates $\left(u_{1}, u_{2}\right)$ on $\mathbb{C P}^{1}$. More correctly stated, the $a_{n}$ are sections of $K_{B}^{-n}$, where $K_{B}$ denotes the canonical bundle of the base $B=\mathbb{C P}^{1}$. Note that $z=0$ defines a section of the elliptic fibration, i.e. the divisor $z=0$ is the base $\mathbb{C P}^{1}$. Completing the square and the cubic term, this so-called Tate form can be written in the so-called Weierstraß form

$$
y^{2}=x^{3}+f_{4} x z^{4}+g_{6} z^{6}
$$

\footnotetext{
1 For base $\mathbb{C} \mathbb{P}^{1}$ the Weierstraßform is sufficient, but for compactifications to six and four dimensions the Tate is very convenient.
} 
To express $f_{4}$ and $g_{6}$ in terms of the $a_{n}$, it is convenient to introduce the objects $b_{2}=a_{1}^{2}+4 a_{2}, b_{4}=$ $a_{1} a_{3}+2 a_{4}$ and $b_{6}=a_{3}^{2}+4 a_{6}$ so that

$$
f_{4}=\frac{1}{48}\left(24 b_{4}-b_{2}^{2}\right), \quad g_{6}=\frac{1}{864}\left(216 b_{6}-36 b_{4} b_{2}+b_{2}^{3}\right) .
$$

Given the Weierstraß form with sections $f_{4}$ and $g_{6}$, the complex structure $\tau$ of the elliptic fiber over a point $\left(u_{1}, u_{2}\right)$ is implicitly given by

$$
j(\tau)=\frac{4\left(24 f_{4}\right)^{3}}{4 f_{4}^{3}+27 g_{6}^{2}},
$$

where indeed the $j$-function appears. Now, the location of the 7-branes should be at the zeros of the denominator

$$
\Delta=4 f_{4}^{3}+27 g_{6}^{2}=-\frac{1}{4} b_{2}^{2}\left(b_{2} b_{6}-b_{4}^{2}\right)-8 b_{4}^{3}-27 b_{6}^{2}+9 b_{2} b_{4} b_{6} .
$$

This is the so-called discriminant of the elliptic fibration and its zeros are mathematically precisely the points where the torus degenerates. Note that $\Delta$ is a polynomial of degree 24 in $\left(u_{1}, u_{2}\right)$, and thus has 24 zeros. These points are the positions of the 24 7-branes on $\mathbb{C P}^{1}$.

So far we assumed that the discriminant has 24 different zeros. However, when some of these zeros coincide the elliptic fibration further degenerates, i.e. certain 2-cycles shrink to zero size. In the M-theory description, M2-branes wrapped on these shrunken 2-cycles provide new massless states, which give rise non-abelian gauge symmetries. In fact there exists a classification by Kodaira [12] of the different types

\begin{tabular}{|c|c|c|c|c|c|c|c|}
\hline $\operatorname{ord}(f)$ & ord $(g)$ & $\operatorname{ord}(\Delta)$ & fiber & singularity & comp. & local geometry & monod. \\
\hline$\geq 0$ & $\geq 0$ & 0 & $\mathrm{I}_{0}$ & smooth & 1 & & $\left(\begin{array}{ll}1 & 0 \\
0 & 1\end{array}\right)$ \\
\hline 0 & 0 & 1 & $\mathrm{I}_{1}$ & dbl. point & 1 & $y^{2}=x^{2}+z$ & $\left(\begin{array}{ll}1 & 1 \\
0 & 1\end{array}\right)$ \\
\hline 0 & 0 & $n$ & $\mathrm{I}_{n}$ & $\mathrm{~A}_{n-1}$ & $n$ & $y^{2}=x^{2}+z^{n}$ & $\left(\begin{array}{ll}1 & n \\
0 & 1\end{array}\right)$ \\
\hline$\geq 1$ & 1 & 2 & II & cusp & 1 & & $\left(\begin{array}{cc}1 & 1 \\
-1 & 0\end{array}\right)$ \\
\hline$\geq 1$ & $\geq 2$ & 3 & III & $\mathrm{A}_{1}$ & 2 & $y^{2}=x^{2}+z^{2}$ & $\left(\begin{array}{cc}0 & 1 \\
-1 & 0\end{array}\right)$ \\
\hline$\geq 2$ & 2 & 4 & IV & $\mathrm{A}_{2}$ & 3 & $y^{2}=x^{2}+z^{3}$ & $\left(\begin{array}{cc}0 & 1 \\
-1 & -1\end{array}\right)$ \\
\hline 2 & 3 & 6 & $\mathrm{I}_{0}^{*}$ & $\mathrm{D}_{4}$ & 5 & $y^{2}=x^{2} z+z^{3}$ & $\left(\begin{array}{cc}-1 & 0 \\
0 & -1\end{array}\right)$ \\
\hline $\begin{array}{r}2 \\
\geq 2\end{array}$ & $\begin{array}{r}\geq 3 \\
3\end{array}$ & $n+6$ & $\mathrm{I}_{n}^{*}$ & $\mathrm{D}_{n+4}$ & $n+5$ & $y^{2}=x^{2} z+z^{n+3}$ & $\left(\begin{array}{cc}-1 & -n \\
0 & -1\end{array}\right)$ \\
\hline$\geq 3$ & 4 & 8 & $\mathrm{IV}^{*}$ & $E_{6}$ & 7 & $y^{2}=x^{3}+z^{4}$ & $\left(\begin{array}{cc}-1 & -1 \\
1 & 0\end{array}\right)$ \\
\hline 3 & $\geq 5$ & 9 & III* $^{*}$ & $E_{7}$ & 8 & $y^{2}=x^{3}+x z^{3}$ & $\left(\begin{array}{cc}0 & -1 \\
1 & 0\end{array}\right)$ \\
\hline$\geq 4$ & 5 & 10 & $\mathrm{II}^{*}$ & $E_{8}$ & 9 & $y^{2}=x^{3}+z^{5}$ & $\left(\begin{array}{cc}0 & -1 \\
1 & 1\end{array}\right)$ \\
\hline
\end{tabular}
such an elliptic fibration over $\mathbb{C P}^{1}$ can degenerate. As shown in table 2, this classification is of the A-D-E type expected for singularities on $K 3$ respectively enhanced gauge symmetries.

Table 2 The Kodaira classification of singular fibers in elliptic surfaces. The local geometry of the elliptic surface around such an A-D-E singularity is modeled in terms of coordinates $(x, y, z) \in \mathbb{C}^{3}$. In the last column the elliptic monodromy of the singular fiber is given in terms of a $S L(2, \mathbb{Z})$-matrix.

Note that in particular the exceptional gauge groups $E_{6}, E_{7}$ and $E_{8}$ can be realized as enhanced gauge symmetries in F-theory. Clearly they cannot be realized by fundamental open strings of the perturbative 
Type IIB string, i.e. not just with $(1,0)$ strings and D7-branes. These enhancements must involve more general $(p, q)$ seven branes and the corresponding string-junctions between them.

To get an idea how this works, we compare the geometric monodromy matrices in table 2 with those listed for stacks of $A, B, C$ branes in table 1 It is evident that for the A-D-E series and the three fiber types II,III,IV we have a perfect match. Moreover, the number of 7-branes is in all cases identical to the vanishing order of the discriminant. One can also show [11] that the string junctions ending on the stacks of branes provide precisely the massless states to fill out the adjoint representation of A-D-E gauge groups. Note that the $D_{n}$ series is realized by the $A^{n} B C$ 7-branes, which indicates that the $B C$ pair can be considered as the non-perturbative description of an $O 7$ plane. Therefore, F-theory goes beyond the perturbative Type IIB orientifolds in that it allows for general $(p, q) 7$-branes and their corresponding $(p, q)$-strings. It is precisely this more general structure which realizes the exceptional gauge groups and as a consequence all their group theoretic consequences, such as matter in spinor representations of $S O(10)$ or the $\mathbf{1 0 1 0 5}_{\mathrm{H}}$ Yukawa coupling for $S U(5)$ GUT models.

\section{F-theory compactifications and the Sen limit}

Finally, to connect to the second lecture on F-theory, let us briefly comment on lower dimensional compactifications of F-theory. Instead of fibering the torus over a complex one-dimensional base, one can consider fibrations over surfaces $B_{2}$ or three-dimensional bases $B_{3}$. Supersymmetry then implies that the total space should either be a Calabi-Yau three-fold (for $B_{2}$ ) or Calabi-Yau four-fold (for $B_{3}$ ). One can still write down a Weierstraß model, where $f_{4}$ and $g_{6}$ are sections of $K_{B}^{-4}$ and $K_{B}^{-6}$. The zeros of the discriminant define complex co-dimension one curves in $B_{2}$ respectively surfaces in $B_{3}$ and give the location of 7-branes. In these cases, it is more convenient to use the Tate form (7) of the elliptic fibration, as there exists a refinement of the Kodaira classification, the so-called Tate algorithm, which allows to determine the gauge group essentially from the vanishing order of the discriminant and the sections $a_{n}$ (see [13] for more details). Now it can however happen that the singularity enhances further where these co-dimension one objects intersect. Similar to intersecting D-branes, this is where additional matter fields are localized.

In the case of an F-theory compactification on a smooth Calabi-Yau four-fold $Y$ a couple of new issues need to be considered. First, one can show that chiral matter only arises on the intersection curve between two 7-branes, if there exists a non-trivial $G_{4}$-form background (M-theory point of view). Second, one finds a non-trivial D3-brane tadpole cancellation condition, which in this case reads

$$
N_{D 3}+\frac{1}{2} \int_{Y} G_{4} \wedge G_{4}=\frac{\chi(Y)}{24}
$$

where $\chi(Y)$ denotes the Euler characteristic of the smooth (appropriately resolved) four-fold $Y$.

One can define a limit in which the string coupling goes to zero almost everywhere on the base. This is the so-called Sen-limit [14], defined by rescaling $a_{3} \rightarrow \varepsilon a_{3}, a_{4}=\varepsilon a_{4}, a_{6}=\varepsilon^{2} a_{6}$ and sending $\varepsilon \rightarrow 0$. In this parameterization one finds

$$
f_{4}=\frac{1}{48}\left(24 \varepsilon b_{4}-b_{2}^{2}\right), \quad g_{6}=\frac{1}{864}\left(216 \varepsilon^{2} b_{6}-36 \varepsilon b_{4} b_{2}+b_{2}^{3}\right) .
$$

so that the discriminant becomes

$$
\Delta=-\frac{\varepsilon^{2}}{4} b_{2}^{2}\left(b_{2} b_{6}-b_{4}^{2}\right)+O\left(\varepsilon^{3}\right) \quad \Rightarrow \quad j(\tau) \simeq \frac{b_{2}^{4}}{\varepsilon^{2}\left(b_{2} b_{6}-b_{4}^{2}\right)} .
$$

Therefore, for $\varepsilon \rightarrow 0$ the Type IIB string coupling constant $g_{s}$ goes to zero almost everywhere except on the locus where $b_{2}$ vanishes. Studying the monodromies one finds a D7-brane on the locus $\left(b_{2} b_{6}-b_{4}^{2}\right)=0$ and an O7-plane where $b_{2}=0$. Therefore, the Sen-limit defines the region in the complex structure moduli space, where F-theory is (almost everywhere) weakly coupled and a perturbative Type IIB orientifold description is justified. 
Acknowledgements I would like to thank Benjamin Jurke and Dieter Lüst for useful comments about the manuscript.

\section{References}

[1] R. Blumenhagen, M. Cvetic, S. Kachru and T. Weigand, Ann. Rev. Nucl. Part. Sci. 59, 269 (2009) arXiv:0902.3251 [hep-th]].

[2] R. Donagi and M. Wijnholt, arXiv:0802.2969 [hep-th].

[3] C. Beasley, J. J. Heckman and C. Vafa, JHEP 0901, 058 (2009) [arXiv:0802.3391 [hep-th]].

[4] C. Beasley, J. J. Heckman and C. Vafa, JHEP 0901, 059 (2009) [arXiv:0806.0102 [hep-th]].

[5] R. Donagi and M. Wijnholt,arXiv:0904.1218 [hep-th].

[6] J. J. Heckman, arXiv:1001.0577 [hep-th].

[7] C. Vafa, Nucl. Phys. B 469, 403 (1996) [arXiv:hep-th/9602022].

[8] F. Denef, arXiv:0803.1194 [hep-th].

[9] R. Blumenhagen, B. Körs, D. Lüst and S. Stieberger, Phys. Rept. 445, 1 (2007) arXiv:hep-th/0610327].

[10] B. R. Greene, A. D. Shapere, C. Vafa and S. T. Yau, Nucl. Phys. B 337, 1 (1990).

[11] M. R. Gaberdiel and B. Zwiebach, Nucl. Phys. B 518, 151 (1998) [arXiv:hep-th/9709013].

[12] K. Kodaira, Annals of Math. 77, 563 (1963).

[13] M. Bershadsky, K. A. Intriligator, S. Kachru, D. R. Morrison, V. Sadov and C. Vafa, Nucl. Phys. B 481, 215 (1996) |arXiv:hep-th/9605200].

[14] A. Sen, Nucl. Phys. B 475, 562 (1996) [arXiv:hep-th/9605150]. 